\section{SAT0641-HPR FACTORS ASSOCIATED WITH COGNITIVE IMPAIRMENT IN KOREAN ADULTS WITH RHEUMATOID ARTHRITIS}

S. Y. Shin ${ }^{1}$, J. H. Lee ${ }^{2}$, B. Y. Youn ${ }^{2}{ }^{1}$ Inje University, Department of Nursing, College of Medicine, Busan, Korea, Rep. of (South Korea); ${ }^{2}$ Inje University llsan Paik Hospital, Division of Rheumatology, Ilsan, Korea, Rep. of (South Korea)

Background: For persons with chronic diseases including rheumatoid arthritis (RA), undamaged cognitive capacity is critical for daily functioning, treatment compliance, and self-management. Disease-specific features of RA such as systemic chronic inflammation or increased comorbid cardiovascular disease (CVD) risk may be closely linked to neurocognitive dysfunction in RA patients [1]. However, the evidence of brain involvement in RA is very rare or even controversial and very little is known about the pathogenic mechanisms of cognitive decline in persons with RA. Objectives: This study explored the prevalence of cognitive impairment in Korean adults with RA using a set of computerized neurocognitive tests and the factors that were significantly associated with cognitive impairment.

Methods: Individuals with RA were recruited by their rheumatologists during follow-up visits at one university hospital in Korea. After getting signed consents, a trained research nurse assessed participants with a range of physical, psychosocial, and biological metrics. Cognitive function was assessed using a set of 6 computerized neurocognitive tests yielding 18 indices covering a range of cognitive domains. Subjects were classified as 'impaired' if they performed $1 S D$ below age-based population norms on each test [2]. The total cognitive function score was calculated by summing the transformed scores, ranging from 0 (no impairment) to 18 (worst impairment). Multiple linear regression analyses were conducted to identify the significant factors influencing cognitive impairment. Results: Sixty five subjects with a mean $( \pm S D)$ age of $61.9( \pm 10.0)$ years were included. $85 \%$ were female, $89 \%$ were married, and $76 \%$ had less 12 years of education. Mean disease activity score (DAS-28) was $2.3( \pm 1.3)$ and mean disease duration was $9.8( \pm 8.7)$ years. Mean functional limitations score $(\mathrm{HAQ})$ was $0.3( \pm 0.5)$ and mean CVD risk factors were $2.3( \pm 1.5)$. Total cognitive function score was $11.1 \pm 4.0(2-18)$. The proportion of persons who were classified as cognitively impaired on each test ranged from $25 \%$ to $92 \%$. The proportion of persons classified as cognitively impaired on the quarter of total subtests (5 or more out of 18 subtests) was $94 \%$. The multivariate regression model was statistically significant and accounted for $39 \%$ of the variance in cognitive impairment $(F=5.26, p<.001)$. Education $(\beta=-0.32, p=0.010)$, family income $(\beta=-0.26$, $p=0.040)$, and cardiovascular disease risk factors $(\beta=0.27, p=0.025)$ were significantly associated with cognitive impairment controlling for other covariates. Conclusion: A significant number of RA patients were cognitively impaired. Less education, less family income, and increased cardiovascular disease risk factors were the significant factors affecting cognitive impairment in RA. The findings of this study suggest that the burden of cognitive impairment in RA patients is significant, and future studies identifying specific etiological contributors to cognitive impairment are warranted.

References:

[1] Wallin, K., Solomon, A., Kåreholt, I., Tuomilehto, J., Soininen, H., \& Kivipelto, M. (2012). Midlife rheumatoid arthritis increases the risk of cognitive impairment two decades later: a population-based study. Journal of Alzheimer's Disease, 31(3), 669-676.

[2] Kozora, E., Ellison, M. C., \& West, S. (2004). Reliability and validity of the proposed American College of Rheumatology neuropsychological battery for systemic lupus erythematosus. Arthritis Care \& Research, 51(5), 810-818.

Acknowledgments: This research was supported by the 2018 Inje University research grant (No.20180148).

Disclosure of Interests: None declared

DOI: 10.1136/annrheumdis-2020-eular.649

\section{SAT0642-HPR CAPTURING THE UNMET NEEDS OF WOMEN WITH SYSTEMIC LUPUS ERYTHEMATOSUS AND IDENTIFYING THE INFORMATION NEEDED IN PHYSICIAN-PATIENT COMMUNICATION}

S. Shoela ${ }^{1}$, A. Beltagy ${ }^{1}$, F. Fayed ${ }^{2}$, M. Morsy ${ }^{1} .{ }^{1}$ Alexandria Faculty of Medicine, Internal Medicine Department, Alexandria, Egypt ${ }^{2}$ Alexandria University Student Hospital, Internal Medicine Department, Alexandria, Egypt

Background: Systemic lupus erythematosus (SLE) is known to affect the reproductive health of female patients in various ways. Identifying the unmet information and needs of women with SLE about the impact of the disease on maternal health, pregnancy, family planning and contraception is of paramount importance.

Objectives: Our aim was to understand the information needs of women with SLE and capture the gaps in the knowledge of reproductive issues.
Methods: We interviewed 284 female patients with SLE in three centers all affiliated to Alexandria Faculty of Medicine, using a 41 multiple-choice based questionnaire about pregnancy counselling, contraception and the use of drugs during pregnancy. The disease knowledge index (DKI) created by Andreoli et el.,(1) was applied to assess the global knowledge and information of patients on the impact of SLE on reproductive health.

Results: Forty percent of patients declared to have performed the last gynecological visit since 3 years, versus $(49.3 \%)$ patients who have done their last visit within a year. 255 patients reported to have received counselling about contraception; 141 of which provided by gynecologists and not rheumatologists. $114(40.1 \%)$ patients have never been asked about the desire to have children Regarding the methods of contraception used, 104 (36.6\%) patients stated that they don't know there are different forms of pills and have never heard of the progestin-only pills. As for the DKI, patients showed proper knowledge about the possibility for SLE women to fall pregnant, have healthy children and the fact that lupus flares up during pregnancy. $118(41.5 \%)$ of the patients didn't know whether children of women with SLE carry a higher risk of having general health problems or not. Also, a great proportion of patients chose "do not know" for the possibility that children could inherit the mother's disease $(49.6 \%)$. Concerning the drugs used during pregnancy, surprisingly, $(34.2 \%)$ patients stated that Hydroquinone shouldn't be used during pregnancy, and (28.9\%) didn't know if it is compatible with pregnancy or not. Nearly half the patients who were interviewed didn't know that Methotrexate, Cyclophosphamide and Mycophenolate mofetil are contraindicated in pregnancy. About $80 \%$ of the patients stated that SLE influenced the number of the family size they desired in various ways and 134 (47.2\%) patients blamed the disease for not being able to take care of their children. Expectedly, SLE impacted patients' marriages in different forms; 27 (9.5\%) of the patients claimed that the disease led to their divorce, $52(18.3 \%)$ explained that their spouses constantly complained about their illnesses, and $19(6.7 \%)$ refused to answer this question. A total of 181 (63.7\%) patients had spontaneous abortions, among which 181 (63.7\%) patients before being diagnosed with SLE, and 134 (74\%) of these abortions occurred in the first trimester.

Conclusion: There is a crucial unmet need for women with SLE, identified as a wide gap in communication about reproductive issues. This is influenced by the quality of physician-patient communication, as well as rheumatologist-obstetrician communication.

References:

[1] Andreoli L, Lazzaroni MG, Carini C, Dall'Ara F, Nalli C, Reggia R, et al. "Disease knowledge index" and perspectives on reproductive issues: A nationwide study on 398 women with autoimmune rheumatic diseases. Joint, bone, spine: revue du rhumatisme. 2019;86(4):475-81.

Acknowledgments: I wish to express my deepest gratitude to Dr.Laura Andreoli and her colleagues for allowing us to use their questionnaire in our study. Disclosure of Interests: None declared

DOI: 10.1136/annrheumdis-2020-eular.4508

\section{SAT0643-HPR NURSE-LED CARE FROM THE PERSPECTIVE OF PEOPLE WITH EARLY RHEUMATOID ARTHRITIS: A QUALITATIVE SYSTEMATIC REVIEW}

A. M. T. Sweeney ${ }^{1}$, C. Mccabe ${ }^{1}$, C. Flurey ${ }^{1}$, J. Robson ${ }^{1}$, A. Berry ${ }^{1}$, P. Richards ${ }^{1}$, M. Ndosi ${ }^{1}{ }^{1}$ Faculty of Health and Applied Sciences, University of the West of England, Bristol, United Kingdom

Background: Nurse-led care has been shown to be clinically effective and cost effective in rheumatoid arthritis (RA) but the role of the nurse in early RA is not well defined. Evidence for processes of care in RA is limited and it is not known how well rheumatology nurse-led clinics meet care needs of people with early RA.

Objectives: The aim of this study was to develop an understanding of rheumatology nurse-led care from the perspective of people with early RA.

Methods: A qualitative systematic review was conducted. The review protocol is published in the International prospective register of systematic reviews.

In March 2019, the following databases were searched: MEDLINE, EMBASE, CINAHL, PsycINFO and OpenGrey. Due to lack of studies in early RA this review included adults with early and established inflammatory arthritis, qualitative studies with data on patients' perspectives of nurse-led care, published in peer-reviewed journals in English between 2010 and 2019. Two reviewers screened titles, abstracts and full texts. Data were extracted and managed in tables. Joanna Briggs Institute Critical Appraisal Checklist was used for quality assessment of the included studies. A thematic synthesis was undertaken using the framework of Thomas and Harden. ${ }^{1}$

Results: The search identified 1034 records. After screening and assessing for eligibility, 8 qualitative studies were included in the review (133 patients), 2 studies included people with early RA. Three main themes were identified (Figure 1). 


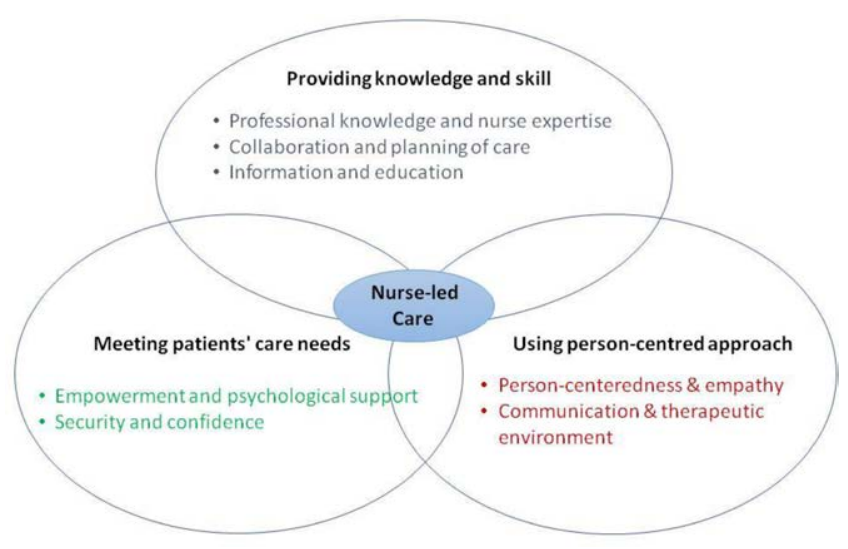

Figure 1. Themes of nurse-led care from the perspective of people with RA

Providing knowledge and skill. This theme delineated rheumatology nursing as providing professional expertise in the planning and delivery of care. The rheumatology nurse-led service included easy access via telephone helpline, consultations with the clinical nurse specialist for assessment of disease activity and care needs, planning of care, disease information and education, supporting self-management, and referral to rheumatologist and the multi-disciplinary team. People with RA highly valued the nurse expertise and specialist knowledge provided at nurse-led clinics. 'She was very good at informing me, so I have only praise for this ... because I have never had it like this before'. (Person with early $R A)$.

Using a person-centred approach. This theme showed nurse-led care using a person-centred approach combined with empathy and good communication skills, which created a good therapeutic environment. People with RA appreciated the person-centeredness, empathy and involvement of the nurse. 'She is very sensitive. She can see if I am feeling bad and comes straight to me and asks: "How are you today?"...You are treated and taken seriously. (Person with early RA).

Meeting patients' care needs. This theme presented nurse-led care as creating a sense of being empowered and psychologically supported in the management of RA and its impact. Nurse-led care made people with RA feel cared for, secure and confident. It added value to rheumatology care and made care complete. 'The thought of sticking a needle into my own stomach... it felt a bit like I would never manage to do that. However, they have been absolutely wonderful here ... and now I can do it myself'. (Person with early RA).

Conclusion: Nurse-led care for people with RA is characterised by provision of rheumatology expertise using a person-centred approach, and patients' holistic care needs are being met. This study found a dearth of literature on perceptions of nurse-led care in people with early RA, which highlights the need for further research in this population.

References:

[1] Thomas J, Harden A. Methods for the thematic synthesis of qualitative research in systematic reviews. BMC Med Res Methodol 2008; 8: 45 .

Disclosure of Interests: Anne-Marie Tetsche Sweeney: None declared, Candy McCabe: None declared, Caroline Flurey: None declared, Joanna Robson: None declared, Alice Berry: None declared, Pamela Richards: None declared, Mwidimi Ndosi Grant/research support from: Bristol Myers Squibb, Consultant of: Janssen, Pfizer

DOI: 10.1136/annrheumdis-2020-eular.502

\section{SAT0644-HPR COMPLIANCE OF BIOLOGIC DISEASES MODIFYING ANTI-RHEUMATIC DRUGS (BDMARDS) WITH SYSTEMIC IMMUNO-INFLAMMATORY RHEUMATIC DISEASES (SIRDS). AN ASSESSMENT OF PATIENTS ADHERENCE AND NON- ADHERENCE CONCERNS.}

R. Thakran ${ }^{1}$, S. Baghel' ${ }^{1}$, C. Messi ${ }^{1}$, V. Kumar' ${ }^{1}$, S. Kapoor ${ }^{1}$, S. Garg ${ }^{1}$, A. Malaviya ${ }^{1}$ on behalf of no. ${ }^{1}$ Indian Spinal Injuries Centre, rheumatology, New Delhi, India

Background: Patients with systemic immunoinflammatory rheumatic diseases (SIRDs) are often treated with bDMARDs when the response to conventional disease-modifying antirheumatic drugs (csDMARDs) is inadequate.There are, however, concerns about non-adherence to bDMARDs among patient. The non-adherence to bDMARDs may be caused by the various factors.

Objectives: 1.The main objective of present study was to find out the cause of discontinuation of bDMARDs

2.To find out the adherence and non-adherence rate for bDMARDs.

3.To identify the factors that are modifiable.
Methods: $\mathbf{8 0 0}$ patients with SIRDs prescribed bDMARDs were interviewed to find out the demographic information, their socioeconomic status, and the disease duration.Additional information gathered included the comorbidities, the time for starting bDMARDs, the route of administration of bDMARDs, beliefs and perceptions about treatment efficacy and side effects if any. This was followed by looking at the adherence of bDMARDs; if they had discontinued then efforts was made to find out the reasons for the same.

Based on these findings the patients were classified into adherent and non-adherent categories. The data were analyzed further for

1.Factors that associated with persistence of bDMARDs.

2.Factors that were associated with discontinuation of bDMARDs.

Results: A total of 800 patients were interviewed that included patients with ankylosing spondylitis $430(52.4 \%)$, rheumatoid arthritis $300(37.7 \%)$, psoriatic arthritis $45(5.2 \%)$, and others $25(0.7 \%)$.On analysis $610(76 \%)$ patient were compliant but 190(24\%) patient had discontinued the bDMARDs on their own. On comparison of both groups

Factors that were significantly related to self-discontinuation were:

- Negative beliefs about biologics (37\%)

- Cost (33\%)

- Reading side-effect profile on Google search (25\%)

- Other co-morbidities (6\%)

Factors that were significantly related to persistence of biologic treatment were:

- Good counseling by rheumatologist and rheumatology nurse (60\%)

- Faith in the treating rheumatologist $(25 \%)$

- Fear of deformities and pain(15\%)

On analysis it was found that a good counseling and clarifying the doubts of the patients regarding bDMARDs before starting the treatment encourages the patient to continue the biologic treatment, especially it allays their doubts about the drug adverse effects.

Conclusion: Despite negative beliefs and misconceptions about bDMARDs, patient non-adherence at our center is not alarming.A positive reinforcement counseling appears to be the most significant factor to overcome the negative belief of patients. The affordability of the biologic treatment however remains a limiting factor in our centre as in other parts of India.

References:

[1] Tamas Koncz,MD,Marta,Pentek,Valentin,Brodszky,Katalin Ersek,MSc Ewaorlewska\&Laszlo Gulasi Volume10,2010 -Issue9 Adherence to biologic DMARD therapies in rheumatoid arthritis

Acknowledgments: no

Disclosure of Interests: None declared

DOI: 10.1136/annrheumdis-2020-eular.3026

\section{SAT0645-HPR MYTHS AND MISCONCEPTION ABOUT THE ILLNESS AND CONVENTIONAL SYNTHETIC DMARDS (CSDMARDS) IN PATIENTS WITH SYSTEMIC IMMUNO- INFLAMMATORY RHEUMATIC DISEASES (SIRDS): A STUDY BY RHEUMATOLOGY NURSE COUNSELOR}

R. Thakran ${ }^{1,2}$, S. Baghel ${ }^{1,3}$, L. Khurshid ${ }^{2}$, S. Kapoor4, S. Garg4, A. Malaviya4on behalf of no. ${ }^{1}$ Indian Spinal Injuries Centre, New Delhi, India; ${ }^{2}$ Indian Spinal Injuries Centre, rheumatology, New Delhi, India; ${ }^{3}$ indian spinal injuries centre, rheumatology, New Delhi, India; ${ }^{1}$ Indian Spinal Injuries Centre, New Delhi, India

Background: Myths and misconceptions about illness and conventional disease modifying anti-rheumatic drugs directly influence adherence to the prescribed treatment. It is estimated that $30-50 \%$ of patients do not adhere to their prescribed treatment due to various reasons where the beliefs of the patients play a crucial role. At our centre we the specialist rheumatology nurse counsel the patients at every visit and try to remove their myths and negative beliefs about the disease as well as the medications.

Objectives: - To explore the common myths and misconceptions of regarding their disease and regarding the csDMARDs.

- To assess the efficacy of counseling in allaying their unfounded fear.

Methods: A total of 450 patients with SIRDs at least 3 times attended the rheumatology out-patient clinic on csDMARDs were enrolled to complete a questionnaire that, besides demographic information, socio-economic status, and co- morbidities, had the following questions:

1. Self reported adherence to medication

2. Misbelieves regarding food items

3. What kind of health-provider was consulted at the onset of the symptoms

4. Their belief/knowledge regarding:

A. The need for physiotherapy.

B. Life style modification requirement

C. About osteoarthritis

D. Medication requirement during remission

E. Pregnancy and DMARDs

$F$. The need of vaccination 